\title{
TRENDS IN ANTIMICROBIAL RESISTANCE OF URINARY TRACT PATHOGENS IN CHILDREN IN CRETE, GREECE: A SINGLE CENTRE EXPERIENCE
}

I. Kalaitzidou ${ }^{1}$, G. Vlachaki ${ }^{1}$, M. Anatoliotaki ${ }^{1}$, F. Ladomenou

${ }^{1}$ Venizeleio General Hospital, Paediatrics, Heraklion, Greece

\section{Background}

Urinary tract infection (UTI) constitutes a common cause of antibiotic use in childhood. Nowadays, there is a growing concern about increasing resistance to antibiotics and emergence of extended spectrum beta lactamase (ESBL) producing uropathogens.

The aim of the present study was to report the most common UTI pathogens and their antimicrobial resistance patterns among children in Crete and compare the results in the region with those reported ten years ago.

\section{Methods}

This 5-year (2012-2016) retrospective study records the uropathogens and their susceptibility patterns among children 30 days to 16 years old, who were discharged with the diagnosis of UTI from a District General Hospital in Crete. After identification of the bacteria, resistance patterns and presence of extended spectrum beta lactamases (ESBLs) were detected. $\underline{\text { Results }}$

UTI was diagnosed in 284 children $(62.0 \%$ female; mean age 26 months).

The occurrence rates of isolated pathogens were:

Escherichia coli (E. coli) $75.4 \%$

Klebsiella spp $9.15 \%$

Proteus spp 5.28\%

Enterobacter spp $3.87 \%$

Pseudomonas aeruginosa $3.52 \%$ Other $2,78 \%$

Uropathogens responsible for community-acquired UTIs in pediatric population in Crete, 2012-2016

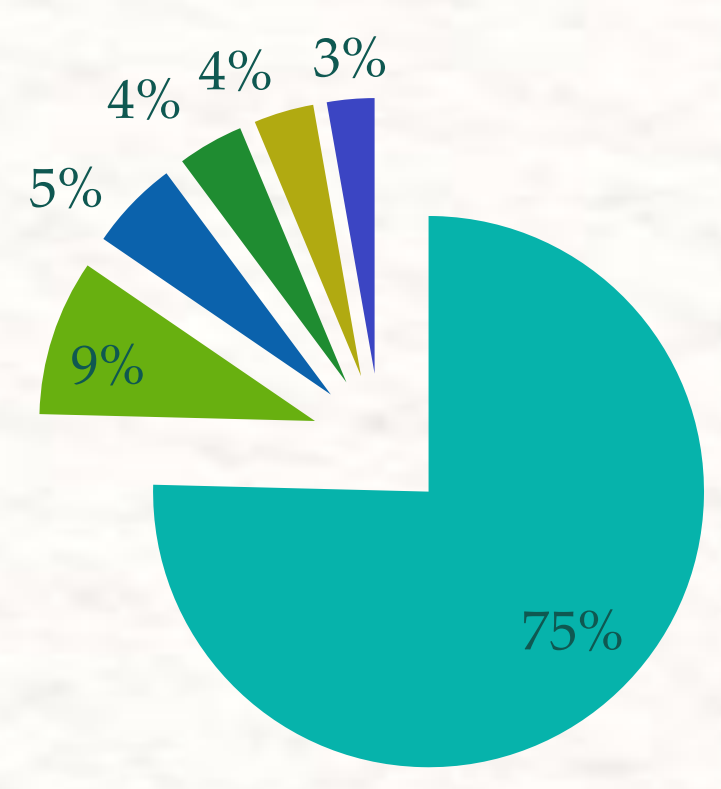

Escherichia coli

- Klebsiella spp

— Proteus spp

Enterobacter spp

Pseudomonas aeruginosa

- Other

\section{Antimicrobial resistant patterns of E.coli}

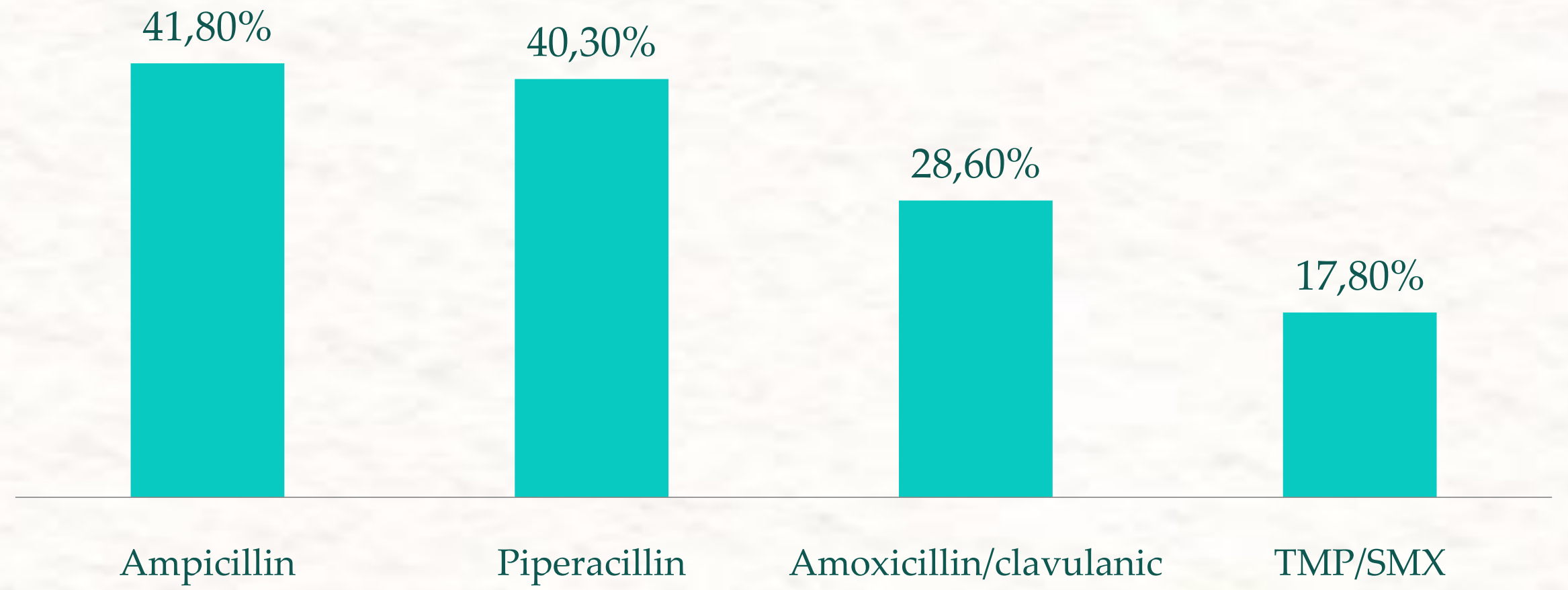

Antimicrobial resistance of $E$. coli was most common to: ampicillin $(41.8 \%)$

piperacillin $(40.3 \%)$

amoxicillin/clavulanic (28.6\%) trimethoprim-sulfamethoxazole (TMP-SMX) (17.8\%)

$4.20 \%$ of E. coli strains were ESBLproducing.

Resistance to ampicillin, TMP-SMX and piperacillin was noted for $46.4 \%, 16.9 \%$ and $46.5 \%$ of the total uropathogens, respectively, making these agents inappropriate for empirical treatment of febrile UTI in our region.
On the other hand, more than $90 \%$ of microorganisms were susceptible to ceftriaxone/cefotaxime cefuroxime, and piperacillin/tazobactam, which seem to be effective for treatment of paediatric UTIs.

\section{Conclusions}

The antibiotic resistance, although decreasing, is still high in hospitalized children with UTIs in the study area. 